\title{
GENERALIZED COMMUTING MAPS ON THE SET OF SINGULAR MATRICES*
}

\author{
WILLIAN FRANCA ${ }^{\dagger}$ AND NELSON LOUZA ${ }^{\dagger}$
}

\begin{abstract}
Let $M_{n}(\mathbb{K})$ be the ring of all $n \times n$ matrices over a field $\mathbb{K}$. In the present paper, additive mappings $G$ : $M_{n}(\mathbb{K}) \rightarrow M_{n}(\mathbb{K})$ such that $[[G(y), y], y]=0$ for all singular matrix $y$ will be characterized. Precisely, it will be proved that $G(x)=\lambda x+\mu(x)$ for all $x \in M_{n}(\mathbb{K})$, where $\lambda \in \mathbb{K}$ and $\mu$ is a central map. As an application, the description is given of all additive maps $g: M_{n}(\mathbb{K}) \rightarrow M_{n}(\mathbb{K})$ such that $\sum_{k_{1}, k_{2}, k_{3}=1}^{m}\left[\left[g\left(y^{k_{1}}\right), y^{k_{2}}\right], y^{k_{3}}\right]=0$ for all singular matrices $y \in M_{n}(\mathbb{K})$, where
\end{abstract} $m \in \mathbb{N}^{*}$.

Key words. Commuting maps, Generalized commuting maps, Functional identities, Singular matrices.

AMS subject classifications. 16R60, 15A99.

1. Introduction. Let $R$ be a ring with center $Z$. A mapping $T: R \rightarrow R$ is called centralizing (resp., commuting) on a subset $H$ of $R$ if $[T(h), h]=T(h) h-h T(h) \in Z$ (resp., [T(h), h] $=0$ ) for all $h \in H$. In [2], Brešar proved, in the case that $R$ is a prime ring, that every additive map $f$ which is centralizing on $R$ has the following standard form:

$$
f(r)=\lambda r+\mu(r) \text { for all } r \in R,
$$

where $\lambda$ lies in the extended centroid $C$ of $R$ and $\mu$ is an additive map from $\mathrm{R}$ into $C$.

In [1], Brešar showed that if $G$ is an additive map of a prime ring $R$ of characteristic not 2 such that $[[G(r), r], r]=0$ for all $r \in R$, then $G$ is commuting on $R$, that is, $G$ has the form (1.1).

The first results on commuting mappings on subsets of matrices that are not closed under addition have appeared in the papers [3, 4]. Basically, it was proved that if the characteristic of a field is zero or strictly greater than 3 , then the only possible additive maps which are either commuting on the set of invertible matrices (resp., singular matrices) or commuting on $R_{k}=$ \{matrices that have rank $k$ \} (for $k>1$ ) are the standard ones.

The case $k=1$ is exceptional. In 2013, the first author [4] provided an example of an additive map which is commuting on $R_{1}$ that does not have the standard form (1.1). Later, in 2016, Franca [9] found the description of all additive maps which are commuting on $R_{1}$. Recently, in [8], we have shown that if we replace $\mathbb{K}$ with a noncommutative division ring $\mathbb{D}$, then an additive map $G: M_{n}(\mathbb{D}) \rightarrow M_{n}(\mathbb{D})$ that is commuting on the set of rank-1 matrices has the standard form (1.1).

To see more results related to functional identities on some subsets which are not closed under addition, we recommend the papers [5, $6,7,10,11]$.

${ }^{*}$ Received by the editors on June 18, 2019. Accepted for publication on October 21, 2019. Handling Editor: Raphael Loewy. Corresponding Author: Willian Franca.

$\dagger$ Department of Mathematics, Federal University of Juiz de Fora, Juiz de Fora, MG - Brazil (wilian.franca@ufjf.edu.br, nelson.louza@ufjf.edu.br). 
2. The main result. Throughout this work $M_{n}(\mathbb{K})$ will denote the ring of all $n \times n$ matrices over a field $\mathbb{K}$ whose characteristic is either zero or greater than 2 . We set $\Omega=\{1, \ldots, n\}$. For index sets $\Omega_{1}, \Omega_{2} \subset \Omega$, we denote by $A\left[\Omega_{1}, \Omega_{2}\right]$ the (sub)matrix of entries that lie in the rows of $A$ indexed by $\Omega_{1}$ and the columns of $A$ indexed by $\Omega_{2}$. Furthermore, we represent by $\bar{\Omega}_{1}$ the complement of $\Omega_{1}$ in $\Omega$.

For each $r, s \in \Omega$, we write $a_{r s}$ to represent the $(r, s)$-entry of a matrix $A \in M_{n}(\mathbb{K})$, where $A=$ $\sum_{r, s=1}^{n} a_{r s} E_{r s}$. In this section, we fix an additive map $G: M_{n}(\mathbb{K}) \rightarrow M_{n}(\mathbb{K})$. Since $G\left(\alpha E_{p q}\right) \in M_{n}(\mathbb{K})$ for each $p, q \in \Omega$, and $\alpha \in \mathbb{K}$, we can write $G\left(\alpha E_{p q}\right)=\sum_{r, s=1}^{n} a_{r s} E_{r s}$, where $a_{r s}=G\left(\alpha E_{p q}\right)_{r s}$.

Now, we will state our first result:

Proposition 2.1. Let $n \in \mathbb{N}(n \geq 2)$ and $G: M_{n}(\mathbb{K}) \rightarrow M_{n}(\mathbb{K})$ be an additive map. Consider the following elements:

(a) $N=\beta E_{i j}$;

(b) $N=\alpha E_{i i}+\theta E_{j j}+\beta E_{i j}+\gamma E_{\xi \xi}$;

(c) $N=\beta E_{i j}+\alpha E_{j \xi}$;

(d) $N=E_{i j}+E_{j \xi}+E_{\xi i}$.

Assume that $[[G(N), N], N]=0$ for all $i, j, \xi \in \Omega$, and for all $\alpha, \beta, \theta, \gamma \in \mathbb{K}$. Then $G$ has the form (1.1).

The proof of the Proposition 2.1 will be divided in a series of technical lemmas in order to make it more transparent. From now on, let $G$ be a mapping as in Proposition 2.1.

Before starting the proofs, we will make a short and relevant observation:

Remark 2.2. Notice that $[[G(N), N], N]=0$ is equivalent to $[G(N), N]$ is in the centralizer of $N$.

Lemma 2.3. $G\left(\beta E_{i i}\right)$ is diagonal for each $i \in \Omega$ and $\beta \in \mathbb{K}$.

Proof. This is clear for $\beta=0$. Let us assume $\beta \neq 0$. Take $N=\beta E_{i i}(N$ has the form $(a)$ for $i=j)$. Remember that each matrix $M$ in the centralizer of $N$ has $M[\overline{[i\}}, i]=0$ and $M[i, \overline{\{i\}}]=0$. Take $r, t \in \Omega$, and $s \in \overline{\{i\}}$. Recall that $[G(N), N]$ belongs to the centralizer of $N$, that is, $[G(N), N]=\xi_{i i} E_{i i}+\sum_{\substack{k, l=1 \\ k, l \neq i}}^{n} \xi_{k l} E_{k l}$. So,

$$
\begin{aligned}
& E_{r s} G(N) E_{i t}=E_{r s}\left[G(N) E_{i i}-E_{i i} G(N)\right] E_{i t}=E_{r s}[G(N), N] E_{i t} \\
& =E_{r s}\left(\xi_{i i} E_{i i}+\sum_{\substack{k, l=1 \\
k, l \neq i}}^{n} \xi_{k l} E_{k l}\right) E_{i t}=\left(\sum_{\substack{l=1 \\
l \neq i}}^{n} \xi_{s l} E_{r l}\right) E_{i t}=0 .
\end{aligned}
$$

Hence,

$$
E_{r s} G(N) E_{i t}=0 .
$$


Therefore,

$$
G\left(\beta E_{i i}\right)[\overline{\{i\}},\{i\}]=0 \text { for all } i \in \Omega
$$

By a similar argument, we see that

$$
G\left(\beta E_{i i}\right)[\{i\}, \overline{\{i\}}]=0 \quad \text { for all } i \in \Omega
$$

Next, take $j \in \overline{\{i\}}$ and consider $N_{1}=\beta E_{i i}+\beta E_{j j}\left(N_{1}\right.$ has the form $\left.(b)\right)$. Remember that each matrix $M$ in the centralizer of $N_{1}$ has $M[\overline{\{i, j\}},\{i, j\}]=0$ and $M[\{i, j\}, \overline{\{i, j\}}]=0$. Since, $\left[G\left(N_{1}\right), N_{1}\right]$ lies in the centralizer of $N_{1}$, we have

$$
G\left(\beta E_{i i}+\beta E_{j j}\right)_{j k}=0 \text { for all } k \in \overline{\{i, j\}} .
$$

Using that the off diagonal entries of $G\left(\beta E_{j j}\right)$ in the $j$-th row (resp., $j$-th column) are zero, and that $G$ is additive, we conclude that the $(j, k)$ entry of $G\left(\beta E_{i i}\right)$ is equal to zero whenever $k \in \overline{\{i, j\}}$. Combined with the above, and allowing $j$ vary over $\overline{\{i\}}$, we conclude that $G\left(\beta E_{i i}\right)$ is a diagonal matrix.

Lemma 2.4. Let $i \in \Omega$ and $j \in \overline{\{i\}}$. Then, $G\left(\beta E_{i j}\right)$ is the sum of a diagonal matrix and a multiple of $E_{i j}$.

Proof. This is clear if $\beta=0$. Let us take $\alpha \in \mathbb{K} \backslash\{0, \beta\}$. Set $N=\alpha E_{i i}+\alpha E_{j j}+\beta E_{i j}$ (using (b)). It can be derived directly from the equation $\left[\left[G\left(\beta E_{i j}\right), \beta E_{i j}\right], \beta E_{i j}\right]=0$ that $G\left(\beta E_{i j}\right)_{j i}=0$. So, if $n=2$ we have established the claim. Otherwise, take $s, t \in \overline{\{i, j\}}$. Remember that each matrix $M$ in the centralizer of $N$ has $M[\overline{[i, j\}},\{i, j\}]=0, M[\{i, j\}, \overline{\{i, j\}}]=0, M_{j i}=0$ and $M_{i i}=M_{j j}$.

So,

$$
[G(N), N]=a_{i j} E_{i j}+\sum_{k=1}^{n} a_{k k} E_{k k}+\sum_{k, l \in \overline{\{i, j\}}} a_{k l} E_{k l} .
$$

On the other hand, taking into account that $G\left(\alpha E_{i i}\right)$ and $G\left(\alpha E_{j j}\right)$ are diagonal, we have

$$
\begin{aligned}
{[G(N), N]=} & {\left[G\left(\alpha E_{i i}\right), \beta E_{i j}\right]+\left[G\left(\alpha E_{j j}\right), \beta E_{i j}\right]+\left[G\left(\beta E_{i j}\right), \alpha E_{i i}\right]+\left[G\left(\beta E_{i j}\right), \alpha E_{j j}\right] } \\
& +\left[G\left(\beta E_{i j}\right), \beta E_{i j}\right] \\
= & \epsilon E_{i j}+\left[G\left(\beta E_{i j}\right), \alpha E_{i i}\right]+\left[G\left(\beta E_{i j}\right), \alpha E_{j j}\right]+\left[G\left(\beta E_{i j}\right), \beta E_{i j}\right]
\end{aligned}
$$

for some $\epsilon \in \mathbb{K}$. After multiplying $[G(N), N]$ by $E_{i t}$ on the left, we arrive at

$$
\begin{aligned}
E_{i t}[G(N), N] & =\alpha\left(E_{i t} G\left(\beta E_{i j}\right) E_{i i}\right)+\alpha\left(E_{i t} G\left(\beta E_{i j}\right) E_{j j}\right)+\beta\left(E_{i t} G\left(\beta E_{i j}\right) E_{i j}\right) \\
& =\alpha\left(G\left(\beta E_{i j}\right)_{t i} E_{i i}+G\left(\beta E_{i j}\right)_{t j} E_{i j}\right)+\beta G\left(\beta E_{i j}\right)_{t i} E_{i j} \\
& =E_{i t}[G(N), N] \stackrel{(2.2)}{=} a_{t t} E_{i t}+\sum_{l \in\{i, j\}} a_{t l} E_{i l} .
\end{aligned}
$$

Then, after comparing the two last lines in the above equality, we conclude that

$$
G\left(\beta E_{i j}\right)_{t i}=G\left(\beta E_{i j}\right)_{t j}=0 \text { for all } t \in \overline{\{i, j\}} .
$$


On the other hand, after multiplying $[G(N), N]$ by $E_{s i}$ on the right and proceeeding similarly as we did before, we see that

$$
\begin{aligned}
{[G(N), N] E_{s i} } & =-\alpha\left(E_{i i} G\left(\beta E_{i j}\right) E_{s i}+E_{j j} G\left(\beta E_{i j}\right) E_{s i}\right)-\beta\left(E_{i j} G\left(\beta E_{i j}\right) E_{s i}\right) \\
& =-\alpha\left(G\left(\beta E_{i j}\right)_{i s} E_{i i}+G\left(\beta E_{i j}\right)_{j s} E_{j i}\right)-\beta G\left(\beta E_{i j}\right)_{j s} E_{i i} \\
& \stackrel{(2.2)}{=} a_{s s} E_{s i}+\sum_{k \in \overline{\{i, j\}}} a_{k s} E_{k i} .
\end{aligned}
$$

So,

$$
G\left(\beta E_{i j}\right)_{i s}=G\left(\beta E_{i j}\right)_{j s}=0 \text { for all } s \in \overline{\{i, j\}} \text {. }
$$

Therefore,

$$
G\left(\beta E_{i j}\right)[\{i, j\}, \overline{\{i, j\}}]=0, \quad G\left(\beta E_{i j}\right)[\overline{\{i, j\}},\{i, j\}]=0, \quad \text { and } G\left(\beta E_{i j}\right)_{j i}=0
$$

Now, take $\xi \in \overline{\{i, j\}}, \gamma \in \mathbb{K} \backslash\{\alpha, 0\}$ and consider $N_{1}=\alpha E_{i i}+\alpha E_{j j}+\beta E_{i j}++\gamma E_{\xi \xi}$. The additivity of $G$ combined with (2.3) and the previous lemma allow us to conclude that $G\left(N_{1}\right)[\{i, j\}, \overline{\{i, j\}}]=0$, $G\left(N_{1}\right)[\overline{\{i, j\}},\{i, j\}]=0$, and $G\left(N_{1}\right)_{j i}=0$. In particular, $G\left(N_{1}\right)_{i, \xi}=G\left(N_{1}\right)_{j, \xi}=G\left(N_{1}\right)_{\xi, i}=G\left(N_{1}\right)_{\xi, j}=0$. Now, we will show that $G\left(N_{1}\right)_{k \xi}=G\left(N_{1}\right)_{\xi l}=0$ for all $k, l \in \overline{\{i, j, \xi\}}$. Indeed, note that $G\left(N_{1}\right)$ can be written as the following:

$$
G\left(N_{1}\right)=\sum_{k=1}^{n} a_{k k} E_{k k}+a_{i j} E_{i j}+\sum_{k, l \in \overline{\{i, j\}}} a_{k l} E_{k l} .
$$

Then,

$$
\begin{aligned}
{\left[G\left(N_{1}\right), N_{1}\right]=} & {\left[G\left(N_{1}\right), \alpha E_{i i}\right]+\left[G\left(N_{1}\right), \alpha E_{j j}\right]+\left[G\left(N_{1}\right), \beta E_{i j}\right]+\left[G\left(N_{1}\right), \gamma E_{\xi \xi}\right] } \\
= & {\left[a_{i j} E_{i j}, \alpha E_{i i}\right]+\left[a_{i j} E_{i j}, \alpha E_{j j}\right]+\sum_{k=1}^{n}\left[a_{k k} E_{k k}, \beta E_{i j}\right] } \\
& +\sum_{k, l \in \overline{\{i, j\}}}\left[a_{k l} E_{k l}, \gamma E_{\xi \xi}\right] \\
= & -\alpha a_{i j} E_{i j}+\alpha a_{i j} E_{i j}+\beta\left(a_{i i}-a_{j j}\right) E_{i j}+\sum_{k, l \in \overline{\{i, j\}}}\left[a_{k l} E_{k l}, \gamma E_{\xi \xi}\right] \\
= & \beta\left(a_{i i}-a_{j j}\right) E_{i j}+\gamma\left(\sum_{k \in \overline{\{i, j\}}} a_{k \xi} E_{k \xi}-\sum_{l \in \overline{\{i, j\}}} a_{\xi l} E_{\xi l}\right) .
\end{aligned}
$$

Remember that $\left[G\left(N_{1}\right), N_{1}\right][\{\xi\}, \overline{\{\xi\}}]=0$ and $\left[G\left(N_{1}\right), N_{1}\right][\overline{\{\xi\}},\{\xi\}]=0$, since $\left[G\left(N_{1}\right), N_{1}\right]$ belongs to the centralizer of $N_{1}$. Then, $G\left(N_{1}\right)_{k \xi}=G\left(N_{1}\right)_{\xi l}=0$ for all $k, l \in \overline{\{i, j, \xi\}}$.

Hence, all off-diagonal entries in row or column $\xi$ of $G\left(N_{1}\right)$ are equal to zero. As $G\left(\alpha E_{i i}\right), G\left(\alpha E_{j j}\right)$ and $G\left(\gamma E_{\xi \xi}\right)$ are diagonal and $G$ is additive, we conclude that all off-diagonal entries in row or column $\xi$ of $G\left(\beta E_{i j}\right)$ are zero. Now, letting $\xi$ vary over $\overline{\{i, j\}}$ the claim is established. 
Lemma 2.5. Let $i \in \Omega$ and $j \in \overline{\{i\}}$. Then, there is a field element $\lambda_{i j}$ not depending on $\beta$ such that $G\left(\beta E_{i j}\right)$ is the sum of a scalar matrix and $\lambda_{i j} \beta E_{i j}$. Besides $\lambda_{i j}=\lambda_{j i}$.

Proof. Let $\alpha, \beta \in \mathbb{K}^{*}$ and consider $N=\beta E_{i j}+\alpha E_{j i}$ (using $(c)$ with $\xi=i$ ). By the additivity of $G$ and the previous lemma, we conclude that

$$
G(N)=G\left(\beta E_{i j}\right)+G\left(\alpha E_{j i}\right)=\sum_{k=1}^{n} a_{k k} E_{k k}+a_{i j} E_{i j}+a_{j i} E_{j i} .
$$

So,

$$
\begin{aligned}
{[G(N), N]=} & {\left[G(N), \beta E_{i j}\right]+\left[G(N), \alpha E_{j i}\right] } \\
= & \sum_{k=1}^{n}\left[a_{k k} E_{k k}, \beta E_{i j}\right]+\left[a_{i j} E_{i j}, \beta E_{i j}\right]+\left[a_{j i} E_{j i}, \beta E_{i j}\right]+\sum_{k=1}^{n}\left[a_{k k} E_{k k}, \alpha E_{j i}\right] \\
& +\left[a_{i j} E_{i j}, \alpha E_{j i}\right]+\left[a_{j i} E_{j i}, \alpha E_{j i}\right] \\
= & \beta\left(a_{i i}-a_{j j}\right) E_{i j}+a_{j i} \beta\left(E_{j j}-E_{i i}\right)+\alpha\left(a_{j j}-a_{i i}\right) E_{j i}+\alpha a_{i j}\left(E_{i i}-E_{j j}\right) .
\end{aligned}
$$

Therefore,

$$
\begin{aligned}
0= & {\left[[G(N), N], \beta E_{i j}+\alpha E_{j i}\right]=\left[[G(N), N], \beta E_{i j}\right]+\left[[G(N), N], \alpha E_{j i}\right] } \\
= & \beta\left\{a_{j i} \beta\left(-2 E_{i j}\right)+\alpha\left(a_{j j}-a_{i i}\right)\left(E_{j j}-E_{i i}\right)+\alpha a_{i j}\left(2 E_{i j}\right)\right\} \\
& +\alpha\left\{\beta\left(a_{i i}-a_{j j}\right)\left(E_{i i}-E_{j j}\right)+a_{j i} \beta\left(2 E_{j i}\right)+\alpha a_{i j}\left(-2 E_{j i}\right)\right\} .
\end{aligned}
$$

So,

$$
2 \beta\left(-a_{j i} \beta+\alpha a_{i j}\right) E_{i j}=0,
$$

and

$$
2 \beta \alpha\left(a_{j j}-a_{i i}\right) E_{j j}=0 .
$$

Thus, $\alpha G(N)_{i j}=\beta G(N)_{j i}$ and $G(N)_{i i}=G(N)_{j j}$ for all $\alpha, \beta \in \mathbb{K}^{*}$, where $N=\beta E_{i j}+\alpha E_{j i}$. Once again, using the previous lemma and the additivity of $G$, we see

$$
\alpha G\left(\beta E_{i j}\right)_{i j}=\beta G\left(\alpha E_{j i}\right)_{j i} \text { for all } \alpha, \beta \in \mathbb{K}^{*},
$$

and

$$
G\left(\beta E_{i j}\right)_{i i}+G\left(\alpha E_{j i}\right)_{i i}=G\left(\beta E_{i j}\right)_{j j}+G\left(\alpha E_{j i}\right)_{j j} \text { for all } \alpha, \beta \in \mathbb{K}^{*}
$$

Fix $\alpha \in \mathbb{K}^{*}$ and let $\beta$ vary over $\mathbb{K}^{*}$ in (2.4). Then,

$$
\frac{G\left(\beta E_{i j}\right)_{i j}}{\beta}=\frac{G\left(\alpha E_{j i}\right)_{j i}}{\alpha}=\lambda_{i j}, \quad \text { for all } \beta \in \mathbb{K}^{*}, \text { where } \lambda_{i j} \in \mathbb{K} .
$$

Hence, $G\left(\beta E_{i j}\right)_{i j}=\beta \lambda_{i j} \forall \beta \in \mathbb{K}^{*}$. Similarly, we conclude that $G\left(\alpha E_{j j}\right)_{j i}=\alpha \lambda_{j i}$ for all $\alpha \in \mathbb{K}^{*}$. Besides, $\lambda_{i j}=\lambda_{j i}$ (note that these equalities hold for $\alpha=0$ and $\beta=0$ ). 
From (2.5), we obtain that $G\left(\beta E_{i j}\right)_{i i}-G\left(\beta E_{i j}\right)_{j j}=G\left(\alpha E_{j i}\right)_{j j}-G\left(\alpha E_{j i}\right)_{i i}$ for all $\alpha, \beta \in \mathbb{K}^{*}$. Fixing $\alpha \in \mathbb{K}^{*}$, and letting $\beta$ vary over $\mathbb{K}^{*}$, we see that

$$
G\left(\beta E_{i j}\right)_{i i}-G\left(\beta E_{i j}\right)_{j j}=v, \text { for all } \beta \in \mathbb{K}^{*} \text {, where } v \in \mathbb{K} .
$$

Take $\beta_{1}, \beta_{2} \in \mathbb{K}^{*}$ such that $\beta_{1}+\beta_{2} \in \mathbb{K}^{*}$. Note

$$
\begin{aligned}
v & \left.=G\left(\left(\beta_{1}+\beta_{2}\right) E_{i j}\right)_{i i}-G\left(\beta_{1}+\beta_{2}\right) E_{i j}\right)_{j j} \\
& =G\left(\beta_{1} E_{i j}\right)_{i i}+G\left(\beta_{2} E_{i j}\right)_{i i}-G\left(\beta_{1} E_{i j}\right)_{j j}-G\left(\beta_{2} E_{i j}\right)_{j j} \\
& =v+v=2 v .
\end{aligned}
$$

Thus,

$$
G\left(\beta E_{i j}\right)_{i i}=G\left(\beta E_{i j}\right)_{j j} \text { for all } \beta \in \mathbb{K}^{*}
$$

Now, we will show that all diagonal entries of $G\left(\beta E_{i j}\right)$ are equal. Indeed, consider $N_{1}=\beta E_{i j}+\alpha E_{j \xi}$, where $\xi \in \overline{\{i, j\}}$. By the previous lemma and the first part of this proof, we can infer that $G\left(N_{1}\right)=$ $\sum_{k=1}^{n} a_{k k} E_{k k}+\lambda_{i j} \beta E_{i j}+\lambda_{j \xi} \alpha E_{j \xi}$, where $\lambda_{i j}$ (resp., $\lambda_{j \xi}$ ) does not depend on $\beta$ (resp., $\alpha$ ). Observe that

$$
\begin{aligned}
{\left[G\left(N_{1}\right), N_{1}\right]=} & {\left[G\left(N_{1}\right), \beta E_{i j}\right]+\left[G\left(N_{1}\right), \alpha E_{j \xi}\right] } \\
= & \sum_{k=1}^{n}\left[a_{k k} E_{k k}, \beta E_{i j}\right]+\left[\lambda_{i j} \beta E_{i j}, \beta E_{i j}\right]+\left[\lambda_{j \xi} \alpha E_{j \xi}, \beta E_{i j}\right] \\
& +\sum_{k=1}^{n}\left[a_{k k} E_{k k}, \alpha E_{j \xi}\right]+\left[\lambda_{i j} \beta E_{i j}, \alpha E_{j \xi}\right]+\left[\lambda_{j \xi} \alpha E_{j \xi}, \alpha E_{j \xi}\right] \\
= & \beta\left(a_{i i}-a_{j j}\right) E_{i j}+\alpha\left(a_{j j}-a_{\xi \xi}\right) E_{j \xi}+\alpha \beta\left(\lambda_{i j}-\lambda_{j \xi}\right) E_{i \xi} .
\end{aligned}
$$

So,

$$
\begin{aligned}
0= & {\left[\left[G\left(N_{1}\right), N_{1}\right], N_{1}\right]=\left[\beta\left(a_{i i}-a_{j j}\right) E_{i j}+\alpha\left(a_{j j}-a_{\xi \xi}\right) E_{j \xi}+\alpha \beta\left(\lambda_{i j}-\lambda_{j \xi}\right) E_{i \xi}, N_{1}\right] } \\
= & \alpha\left(a_{j j}-a_{\xi \xi}\right)\left[E_{j \xi}, \beta E_{i j}\right]+\lambda \beta\left(\lambda_{i j}-\lambda_{j \xi}\right)\left[E_{i \xi}, \beta E_{i j}\right]+\beta\left(a_{i i}-a_{j j}\right)\left[E_{i j}, \alpha E_{j \xi}\right] \\
& +\alpha \beta\left(\lambda_{i j}-\lambda_{j \xi}\right)\left[E_{i \xi}, \alpha E_{j \xi}\right] \\
= & \alpha\left(a_{\xi \xi}-a_{j j}\right) \beta E_{i \xi}+\beta\left(a_{i i}-a_{j j}\right) \alpha E_{i \xi} .
\end{aligned}
$$

Thus, $\left(a_{i i}-a_{j j}\right)=\left(a_{j j}-a_{\xi \xi}\right)$. Hence, $G\left(N_{1}\right)_{i i}-G\left(N_{1}\right)_{j j}=G\left(N_{1}\right)_{j j}-G\left(N_{1}\right)_{\xi \xi}$. This last equality yields

$$
\begin{aligned}
& \left(G\left(\beta E_{i j}\right)_{i i}+G\left(\alpha E_{j \xi}\right)_{i i}\right)-\left(G\left(\beta E_{i j}\right)_{j j}+G\left(\alpha E_{j \xi}\right)_{j j}\right) \\
& =\left(G\left(\beta E_{i j}\right)_{j j}+G\left(\alpha E_{j \xi}\right)_{j j}\right)-\left(G\left(\beta E_{i j}\right)_{\xi \xi}+G\left(\alpha E_{j \xi}\right)_{\xi \xi}\right) .
\end{aligned}
$$

Employing equation (2.6) (twice), we can deduce that $G\left(\alpha E_{j \xi}\right)_{i i}-G\left(\alpha E_{j \xi}\right)_{j j}=G\left(\beta E_{i j}\right)_{j j}-G\left(\beta E_{i j}\right)_{\xi \xi}$ for all $\alpha, \beta \in \mathbb{K}^{*}$. Repeating an earlier argument, we see that $G\left(\beta E_{i j}\right)_{j j}-G\left(\beta E_{i j}\right)_{\xi \xi}=0$ for all $\xi \in \overline{\{i, j\}}$. Then,

$$
G\left(\beta E_{i j}\right)_{i i} \stackrel{(2.6)}{=} G\left(\beta E_{i j}\right)_{j j}=G\left(\beta E_{i j}\right)_{\xi \xi} \quad \text { for all } \xi \in \overline{\{i, j\}} .
$$

Hence, all diagonal entries of $G\left(\beta E_{i j}\right)$ are equal. 
LEMma 2.6. Let $i \in \Omega$ and $j \in \overline{\{i\}}$. Then, $\lambda_{i j}=\lambda$, that $i s, \lambda_{i j}$ does not depend on $i$ and $j$. In particular, $G\left(\beta E_{i j}\right)-\lambda \beta E_{i j}$ is a scalar matrix, where $\lambda \in \mathbb{K}$.

Proof. Consider $N=E_{i j}+E_{j \xi}+E_{\xi i}$, where $\xi \in \overline{\{i, j\}}$ (using $(d)$ ). Then, $G(N)==c I+\lambda_{i j} E_{i j}+$ $\lambda_{j \xi} E_{j \xi}+\lambda_{\xi i} E_{\xi i}$ for some $c \in \mathbb{K}$. Observe

$$
\begin{aligned}
{[G(N), N]=} & {\left[G(N), E_{i j}+E_{j \xi}+E_{\xi i}\right]=\lambda_{i j}\left(\left[E_{i j}, E_{j \xi}\right]+\left[E_{i j}, E_{\xi i}\right]\right)+\lambda_{j \xi}\left(\left[E_{j \xi}, E_{i j}\right]\right.} \\
& \left.+\left[E_{j \xi}, E_{\xi i}\right]\right)+\lambda_{\xi i}\left(\left[E_{\xi i}, E_{i j}\right]+\left[E_{\xi i}, E_{j \xi}\right]\right) \\
= & \lambda_{i j}\left(E_{i \xi}-E_{\xi j}\right)+\lambda_{j \xi}\left(-E_{i \xi}+E_{j i}\right)+\lambda_{\xi i}\left(E_{\xi j}-E_{j i}\right) \\
= & \left(\lambda_{i j}-\lambda_{j \xi}\right) E_{i \xi}+\left(\lambda_{\xi i}-\lambda_{i j}\right) E_{\xi j}+\left(\lambda_{j \xi}-\lambda_{\xi i}\right) E_{j i} .
\end{aligned}
$$

So,

$$
\begin{aligned}
0= & {[[G(N), N], N]=\left[[G(N), N], E_{i j}+E_{j \xi}+E_{\xi i}\right] } \\
= & \left(\lambda_{i j}-\lambda_{j \xi}\right)\left(\left[E_{i \xi}, E_{i j}\right]+\left[E_{i \xi}, E_{j \xi}\right]+\left[E_{i \xi}, E_{\xi i}\right]\right)+\left(\lambda_{\xi i}-\lambda_{i j}\right)\left(\left[E_{\xi j}, E_{i j}\right]\right. \\
& \left.+\left[E_{\xi j}, E_{j \xi}\right]+\left[E_{\xi j}, E_{\xi i}\right]\right)+\left(\lambda_{j \xi}-\lambda_{\xi i}\right)\left(\left[E_{j i}, E_{i j}\right]+\left[E_{j i}, E_{j \xi}\right]+\left[E_{j i}, E_{\xi i}\right]\right) .
\end{aligned}
$$

Therefore,

$$
\begin{aligned}
0= & \left(\left(\lambda_{i j}-\lambda_{j \xi}\right)-\left(\lambda_{j \xi}-\lambda_{\xi i}\right)\right) E_{i i}+\left(\left(\lambda_{j \xi}-\lambda_{\xi i}\right)-\left(\lambda_{\xi i}-\lambda_{i j}\right)\right) E_{j j} \\
& +\left(\left(\lambda_{\xi i}-\lambda_{i j}\right)-\left(\lambda_{i j}-\lambda_{j \xi}\right)\right) E_{\xi \xi} .
\end{aligned}
$$

Thus, we arrive in the following system

$$
\left\{\begin{array}{ccccc}
\lambda_{i j}-2 \lambda_{j \xi}+\lambda_{\xi i} & =0 \\
\lambda_{i j}+\lambda_{j \xi} & -2 \lambda_{\xi i} & = & 0 \\
-2 \lambda_{i j}+\lambda_{j \xi}+\lambda_{\xi i} & = & 0
\end{array} .\right.
$$

Let $A$ be the matrix formed by the coefficients of the above matrix, that is,

$$
A=\left(\begin{array}{ccc}
1 & -2 & 1 \\
1 & 1 & -2 \\
-2 & 1 & 1
\end{array}\right)
$$

Note that after some elementary row operations, the matrix $A$ is equivalent to the following matrix:

$$
B=\left(\begin{array}{ccc}
1 & -2 & 1 \\
0 & 1 & -1 \\
0 & 0 & 0
\end{array}\right)
$$

Then, the solutions are $\lambda_{i j}=\lambda_{j \xi}=\lambda_{\xi i}$ for all distinct $i, j$, and $\xi$. This implies that $\lambda_{i j}$ is independent of $i$ and $j$, since $\lambda_{i j}=\lambda_{j i}\left(\operatorname{using} \lambda_{j i}=\lambda_{\xi j}=\lambda_{i \xi}\right)$. In particular, $G\left(\beta E_{i j}\right)-\lambda \beta E_{i j}$ is a scalar matrix.

Lemma 2.7. Let $i \in \Omega$. Then, $G\left(\alpha E_{i i}\right)-(\lambda \alpha) E_{i i}$ is a scalar matrix, where $\lambda \in \mathbb{K}$ is in accordance with Lemma 2.6. 
Proof. Let $j \in \overline{\{i\}}$. Consider $N=\alpha E_{i i}+\gamma E_{j j}+\beta E_{i j}$ (using (b)), where $\alpha, \beta$ and $\gamma \in \mathbb{K}^{*}$ with $\alpha \neq \gamma$. Using the previous lemmas, we know that $G(N)=\sum_{k=1}^{n} a_{k k} E_{k k}++\lambda \beta E_{i j}$. So,

$$
\begin{aligned}
{[G(N), N] } & =\alpha\left[G(N), E_{i i}\right]+\gamma\left[G(N), E_{j j}\right]+\beta\left[G(N), E_{i j}\right] \\
& =\alpha \lambda \beta\left[E_{i j}, E_{i i}\right]+\gamma \lambda \beta\left[E_{i j}, E_{j j}\right]+\beta \cdot \sum_{k=1}^{n} a_{k k}\left[E_{k k}, E_{i j}\right] \\
& =-\alpha \lambda \beta E_{i j}+\gamma \lambda \beta E_{i j}+\beta\left(a_{i i}-a_{j j}\right) E_{i j}=\rho E_{i j} .
\end{aligned}
$$

Then,

$$
\begin{aligned}
{[[G(N), N], N] } & =\rho\left[E_{i j}, N\right]=\rho\left(\alpha\left[E_{i j}, E_{i i}\right]+\gamma\left[E_{i j}, E_{j j}\right]\right) \\
& =\rho\left(-\alpha E_{i j}+\gamma E_{i j}\right)=\rho(\gamma-\alpha) E_{i j}=0 .
\end{aligned}
$$

Thus, $\rho(\gamma-\alpha)=0$. So, $\rho=\beta\left(-\alpha \lambda+\gamma \lambda+\left(a_{i i}-a_{j j}\right)\right)=0$, because $\alpha \neq \gamma$. Furthermore, since $\beta \in \mathbb{K}^{*}$, we see that $a_{i i}+\gamma \lambda=a_{j j}+\alpha \lambda$.

Therefore,

$$
G(N)_{i i}+\gamma \lambda=G(N)_{j j}+\alpha \lambda .
$$

Let us recall that $G$ is additive, $N=\alpha E_{i i}+\gamma E_{j j}+\beta E_{i j}$, and $G\left(\beta E_{i j}\right)_{i i}=G\left(\beta E_{i i}\right)_{j j}$ (by Lemma 2.6). Hence,

$$
G\left(\alpha E_{i i}\right)_{i i}+G\left(\gamma E_{j j}\right)_{i i}+\gamma \lambda=G\left(\alpha E_{i i}\right)_{j j}+G\left(\gamma E_{j j}\right)_{j j}+\alpha \lambda .
$$

Then,

$$
G\left(\alpha E_{i i}\right)_{i i}-G\left(\alpha E_{i i}\right)_{j j}-\lambda \alpha=G\left(\gamma E_{j j}\right)_{j j}-G\left(\gamma E_{j j}\right)_{i i}-\gamma \lambda
$$

Observe that the left (resp., right) hand side of the above equation only depends on $\alpha$ (resp., $\gamma$ ). Letting $\gamma$ vary on $\mathbb{K}^{*}$, we see $H(\alpha)=G\left(\alpha E_{i i}\right)_{i i}-G\left(\alpha E_{i i}\right)_{j j}-\alpha \lambda=v$, where $v \in \mathbb{K}$. Therefore, $G\left(\alpha E_{i i}\right)_{i i}-$ $G\left(\alpha E_{i i}\right)_{j j}-\lambda \alpha=0$, since $H(\alpha)$ is additive. So, $G\left(\alpha E_{i i}\right)_{i i}-\lambda \alpha=G\left(\alpha E_{i i}\right)_{j j}$ for all $\alpha \in \mathbb{K}^{*}$ and $j \in \overline{\{i\}}$. And this allows us to conclude that $G\left(\alpha E_{i i}\right)_{j j}=G\left(\alpha E_{i i}\right)_{\xi \xi}$ for all $j, \xi \in\{i\}$ and $\alpha \in \mathbb{K}^{*}$. Therefore, $G\left(\alpha E_{i i}\right)-(\lambda \alpha) E_{i i}$ is a scalar matrix. And this completes Proposition's 1.1 proof.

Now, we are in a position to prove our main result:

TheOREM 2.8. Let $\mathbb{K}$ be a field whose characteristic is either zero or greater than 2 , and $n \geq 4$. Let $G: M_{n}(\mathbb{K}) \rightarrow M_{n}(\mathbb{K})$ be an additive map such that

$$
[[G(y), y], y]=0 \quad \text { for all singular } y \in M_{n}(\mathbb{K}) .
$$

Then, there exist an element $\lambda \in \mathbb{K}$ and a central map $\mu$ such that

$$
G(x)=\lambda x+\mu(x) \text { for each } x \in M_{n}(\mathbb{K}) .
$$

Proof. The result follows immediately from Proposition 1.1, because if $N$ has one of the froms $(a),(b),(c)$ or $(d)$ then $N$ is singular for all $n \geq 4$. 
As an application, we have:

Corollary 2.9. Let $m, n \in \mathbb{N}^{*}$, where $n \geq 4$. Let $G: M_{n}(\mathbb{K}) \rightarrow M_{n}(\mathbb{K})$ be an additive map. Let us suppose that

$$
\sum_{k_{1}, k_{2}, k_{3}=1}^{m}\left[\left[G\left(y^{k_{1}}\right), y^{k_{2}}\right], y^{k_{3}}\right]=0
$$

for all singular matrices $y \in M_{n}(\mathbb{K})$. If the characteristic of $\mathbb{K}$ is either zero or greater than $3 m-2$ then $G(x)=\lambda x+\mu(x)$ for each $x \in M_{n}(\mathbb{K})$, where $\lambda \in \mathbb{K}$ and $\mu$ is a central map.

Proof. Let us denote by $\mathbb{L}$ the prime field of $\mathbb{K}$. Let $\beta \in \mathbb{L}^{*}$ and $x \in M_{n}(\mathbb{K})$ be a singular matrix. It is clear that $\beta x$ is singular. Besides, note that $G(\beta x)=\beta x$, since $G$ is additive. By (2.7), we have

$$
\begin{aligned}
& \sum_{k_{1}, k_{2}, k_{3}=1}^{m}\left[\left[G\left(\beta^{k_{1}} x^{k_{1}}\right), \beta^{k_{2}} x^{k_{2}}\right], \beta^{k_{3}} x^{k_{3}}\right] \\
= & \beta^{3}[[G(x), x], x]+\sum_{\substack{k_{1}, k_{2}, k_{3}=1 \\
k_{1}+k_{2}+k_{3} \geq 4}}^{m}\left[\left[G\left(\beta^{k_{1}} x^{k_{1}}\right), \beta^{k_{2}} x^{k_{2}}\right], \beta^{k_{3}} x^{k_{3}}\right] \\
= & \beta^{3}[[G(x), x], x]+\beta^{4} R_{4}(x)+\beta^{5} R_{5}(x)+\cdots+\beta^{3 m} R_{3 m}(x)=0 .
\end{aligned}
$$

So,

$$
[[G(x), x], x]+\sum_{i=4}^{3 m} \beta^{i-3} R_{i}(x)=0 \quad \text { for all } \beta \in \mathbb{L}^{*}
$$

Since, $|\mathbb{L}|>3 m-2$, we can choose $\beta_{1}, \beta_{2}, \ldots, \beta_{3 m-2} \in \mathbb{L}^{*}$ pairwise distinct. Hence,

$$
\left(\begin{array}{ccccc}
1 & \beta_{1} & \beta_{1}^{2} & \cdots & \beta_{1}^{3(m-1)} \\
1 & \beta_{2} & \beta_{2}^{2} & \cdots & \beta_{2}^{3(m-1)} \\
\vdots & \vdots & \vdots & & \vdots \\
1 & \beta_{3 m-2} & \beta_{3 m-2}^{2} & \cdots & \beta_{3 m-2}^{3(m-1)}
\end{array}\right)\left(\begin{array}{c}
{[[G(x), x], x]} \\
R_{4}(x) \\
R_{5}(x) \\
\vdots \\
R_{3 m}(x)
\end{array}\right)=\left(\begin{array}{c}
0 \\
0 \\
0 \\
\vdots \\
0
\end{array}\right)
$$

Therefore, from the above system, we can conclude that $[[G(x), x], x]=0$ for all singular matrices $x \in M_{n}(\mathbb{K})$. Now, the result follows from Theorem 2.8 .

Acknowledgments. The authors would like to thank an anonymous referee for suggesting and outlining a more transparent proof throughout a series of lemmas.

\section{REFERENCES}

[1] M. Brešar. On generalization of the notion of centralizing mappings. Proc. Amer. Math. Soc., 114:641-649, 1992.

[2] M. Brešar. Centralizing mappings and derivations in prime rings. J. Algebra, 156:385-394, 1993.

[3] W. Franca. Commuting maps on some subsets of matrices that are not closed under addition. Linear Algebra Appl., 437:388-391, 2012. 
[4] W. Franca. Commuting maps on rank-k matrices. Linear Algebra Appl., 438:2813-2815, 2013.

[5] W. Franca. Commuting traces of multiadditive maps on invertible and singular matrices. Linear Multilinear Algebra, 61:1528-1535, 2013

[6] W. Franca. Commuting traces on invertible and singular operators. Oper. Matrices, 9:305-310, 2015.

[7] W. Franca. Commuting traces of biadditive maps on invertible elements. Comm. Algebra, 44:2621-2634, 2016.

[8] W. Franca. Commuting maps on rank-1 matrices over noncommutative division rings. Comm. Algebra, 45:4696-4706, 2017.

[9] W. Franca. Weakly commuting maps on the set of rank-1 matrices. Linear Multilinear Algebra, 65:475-495, 2017.

[10] C.-K Liu. Centralizing maps on invertible or singular matrices over division rings. Linear Algebra Appl., 440:318-324, 2014.

[11] C.-K. Liu. Strong commutativity preserving maps on some subsets of matrices that are not closed under addition. Linear Algebra Appl., 458:280-290, 2014. 